incidence with cases often arising from cousin marriages of unaffected members, who can be given no assurance that they will not transmit the disorder. On an average one of every four children will be affected. Peroneal muscular atrophy, the cerebromacular degenerations, torsion spasm, albinism, hepatolenticular degeneration, and some myopathies may show this mode of heredity.

Leber's optic atrophy is amongst the disorders which show a sex-linked recessive tendency, being chiefly found in male members of affected families. When not sex-linked, it tends to appear in early adult life in males, but near the menopause in female subjects.

Epilepsy follows no regular genetic pattern, although a positive family history of fits is not infrequent if carefully looked for, and this can be correlated with a high incidence of electroencephalographic abnormalities in the families of epileptics. Additional non-genetic factors undoubtedly play an important part in the actual production of epilepsy. Russell Brain (24), whose review of the genetics of neurological disorders is a mine of valuable information freely drawn on above, states that approximately one in ten of the children of an epileptic is affected by the disorder. In the absence, however, of a family history of fits, the risk of direct transmission appears to be statistically small, though again probably raised by consanguineous parentage.

\title{
REFERENCES
}

(r) RUSSElL, W. R., IRONSIDE, R., MARTIN, J. P., et al. (1939), Pro. Roy. Soc. Med. 32, 865.

(2) STRAUSS, M. B., and MCDONALD, W. J. (I933), J.A.M.A. $100, \mathrm{I}_{2}$.

(3) CAMPBELL, A. C. P., and BIGGART, J. H. (I939), J. Path. and Bact. 48, 245.

(4) UNGLEY, C. C. (1933), J. Neurol. and Psychopath. 14, 15.

5 PAKOZOY, K. (I929), Deutsch. Med. Wchnschr. 55, I 509.

(6) ALPERS, B. J., and PALMER, H. D. (1929), Journ. Nerv. and Ment. Dis. 70, 465.

(7) WILLSON, P., and PREECE, A. A. D. (1932), Arch. Int. Med. 49, 471.

(8) HUBBLE,'D. (1943), B.M.J. 1, I2I.

(9) BROWNE, F. J. (1942), A ntenatal and Postnatal Care (London).

(I0) WILSON, S. A. K. (1940), Neurology (London).

(II) MARTIN, J. P. (194I), B.M.J. 2, $537.112,138$.

(I3) TRAQUÁIR, H. M. (I942), Clinical Perimetry (London).

(14) MOORE, R. F. (I925), Medical Ophthalmology (London).

(15) TIDY, L. (I94I), B.M.J. $1,774$. . 76 . G. L. (1943), Archiv. Neurol. and Psych. 49, 204.

(I7) NERRLINGER (1889), Inaug. Dissert. (Strasbourg).

I8) TURNER, W. A. (I907), Epilepsy, a study (London).

I9) MUSKENS, L. J. J. (1928). Epilepsy, Comparative Pathogenesis, Symptoms and Treatment (London).

(20) VIETS, H. R., et al. (I942), J.A.M.A. 62, 122 .

2I) NAYRAC, P., GERNEZ, L., DUVAL, R. (1939), Gaz. des Hop. 112, 493.

(22) ROQUES, F.' (1928), Journ. Obst. and Gyn. Brit. Emp. 35, I

(23) SHEEHAN, H. L. (1939), Quart. Journ. Med. 8, 277.

(24) BRAIN, R. (1934), in Chances of Morbid Inheritance, ed. C. P. Blacker (London).

\section{DISORDERS OF THE ENDOCRINE GLANDS IN RELATION TO PREGNANCY}

\author{
By S. L. SIMPSON, M.D., F.R.C.P.
}

(Physician, Willesden General Hospital, with charge of Diabetic Clinic; Physician, Princess Louise Kensington Hospital for Children; Consulting Physician, Soho Hospital for Women.)

Pregnancy should not be thought of as a condition affecting only the reproductive glands and organs, even when it is entirely physiological and uncomplicated by disease. Through the interrelationship of the endocrine glands, changes are probably produced in all the endocrine glands, the effects being only temporary in many cases, but in others a residual degree of change persists. The extent of this physiological change depends on what I have termed "the endocrine constitution" of the individual. The reality of such an "endocrine constitution" can be appreciated by observing familial endocrine tendencies, or stigmata, as variants of so-called normality, and more specifically by studying, for example, the effects of bilateral ovariectomy. 
Some women will gain three stone in six months after bilateral ovariectomy, while others will not change weight; some will have almost unbearable vasomotor and nervous symptoms, others will have none. Similarly with pregnancy, some women show minimal endocrine changes, others put on weight during pregnancy, and/or after each successive pregnancy; while others develop or increase pre-existing hirsutism, temporarily or permanently. These are normal changes probably associated with the hypertrophy of the anterior pituitary gland and adrenal cortex, which we know occur in experimental animals during pregnancy. There is an indefinite dividing line between such normal physiological changes and the development of morbid conditions.

\section{Hormone Tests for Pregnancy}

The Ascheim-Zondek (mice) and Friedman (rabbit) tests for pregnancy are well known and commonly used, the former taking 5 days and the latter 24 to 48 hours. A 6-hour test on rats has recently been evolved by Salmon and colleagues (I942). All tests depend upon the high concentration of chorionic gonadotropic hormone in pregnancy urine, and its effect on the ovary.

\section{Ascheim-Zondek test.}

Six infantile mice are used, each weighing from 6-8 grams. A first morning specimen of urine is collected, acidified slightly with dilute acetic acid and $0 \cdot 25$ c.c. are injected subcutaneously twice daily for three successive days. No injections are given on the fourth day, and the mice are killed on the fifth day. If the test is positive, the ovaries, examined by a hand lens, will show several corpora lutea or punctate haemorrhages. A morning specimen of urine contains a higher concentration of hormone than one collected during the day. Some six mice are used, since one or more may die before the test is completed, and occasionally one is unresponsive to the gonadotropic stimulus. The accuracy of the test is 99 per cent. There are on record, however, definite pregnancies that gave more than one completely negative reaction. More than 60 per cent of pregnancies will give a positive result when the patient is only two days overdue, and more than 90 per cent when the patient is ten days overdue. The appearance of a positive test depends upon the nidation of the ovum, and the establishment of a metabolice interchange between the trophoblast and the maternal circulation. The test remains positive throughout pregnancy up to about the tenth day post-partum. A positive test does not mean? that the foetus has remained alive. It only means that the trophoblast has remained alive and the placenta may go on living several months after the foetus has died. If a positive test subsequently gives place to a negative test, this indicates the death of the foetus, but such a test should be repeated and taken in conjunction with the clinical findings. The test will be positive in an ectopic gestation, as long as there is a living chorionic epitheleum. Haemorrhage. from a ruptured tube is considered unlikely in the presence of a persistently negative test. Both hydatid mole and chorio-epithelioma usually give a positive Ascheim-Zondek, and the concentrations of gonadotropic hormone is ten times as high as in normal pregnancy; but a mole may be present with a negative test. The test may remain positive six weeks after the expulsion of a hydatid mole, but if it persists longer than this, or if it becomes positive again after having been negative, a chorioepithelioma should be suspected.

\section{Friedman test.}

Rabbits do not ovulate spontaneously, but only on coitus or pseudocoitus, or if gonadotropic hormone is injected. Immature female rabbits of twelve weeks are used and kept in separate cages for two or more weeks. Then Io c.c. of morning urine are slowly injected into the ear vein of the rabbit and laparotomy, or killing, is carried out 24 to 48 hours later, the latter period of time being more reliable. One or more haemorrhagic follicles are positive of pregnancy. The test is correct in 90 per cent of cases or more, but when in doubt an AscheimZondek test should also be carried out.

\section{Salmon test.}

This test is of recent origin, and has the advantage of giving a result within six hours. Three immature rats (weight 35 to 45 grams, aged 22-25 days) are injected subcutaneously with 2 c.c.s of the night specimen of urine and autopsied at the end of six hours. If the test is positive, the ovaries are enlarged and bright red in appearance, in sharp contrast to the small white or 
faintly pink ovaries of the control animals. This test was positive in 77 out of 78 pregnancy urine tests, and negative in $3 I$ out of $3 I$ non-pregnancy urines.

\section{Parturition and Abortion}

In I929 Knaus showed that the human uterus does not react to pituitrin (containing both oxytocin and vasopressin) during the second part of the menstrual cycle when the corpus luteum is active; and it has been generally held that progesterone tends to inhibit uterine contraction, and that a fall in the level of progesterone may initiate parturition or abortion. Bilateral ovariectomy, however, does not necessarily interfere with pregnancy or parturition in the human subject, and Robson and Illingsworth (I932) were unable to demonstrate any progestational or inhibitory hormone in the four placentas removed at various stages during gestation. Robson (I933) studied the reactivity of the uterus, to oxytocin and vasopressin, removed at various stages of pregnancy and parturition for a variety of reasons. He found that there was an absence of reactivity in the earliest stages, followed by a greater reactivity to vasopressin than oxytocin, and then in the later stages a gradual increase in the reactivity to oxytocin culminating in labour. So that the inherent reactivity of the uterus at various phases of pregnancy is as important as the circulating hormones, unless one assumes that the circulating hormones have produced some change in the uterine muscle which determines its response to oxytocin even after excision. In a further paper Robson (I933) points out that the reactivity of the uterus after parturition falls rapidly, although the luteal secretion must by then have practically ceased. He points out that oestrin, which is secreted by the placenta as well as by the ovary, has a progressively increasing high concentration in the blood during pregnancy, reaching a maximum at parturition, and rapidly decreasing after expulsion of the placenta; and that the concentration of oestrin determines the reactivity of the uterus to oxytocin. This thesis was proved experimentally by Robson and others in later experiments on animals. Falls, Lackner, and Krohn (I936) claimed to prove by an intra-uterine pressure hag, that in the human uterus oestradiol increased and progesterone decreased the sensitivity of the human uterus to an oxytocin stimulus. They also showed that progesterone was effective in counteracting a threatened abortion or a tendency to abortion that could be anticipated from the experience of previous pregnancies. These and subsequent papers by others have met with criticism and some scepticism. Davis, Hamblen, Cuyler and Baptist (I942) have produced some biological data and clinical studies which tend to confirm older theories. They showed that initial low levels of, or fall in, urinary values for sodium pregnanediol glucoronide were associated with abortion; and that progesterone was effective in the prophyllaxis and treatment of threatened abortion. They injected 5 to Io $\mathrm{mg}$. of progesterone daily or twice weekly, but found that anhydrohydroxy progesterone given orally in daily doses varying from 20 to $60 \mathrm{mg}$. was equally effective. Falls and others (I942) also found progesterone effective in threatened abortion, and in cases of surgical emergency necessitating laparotomy in which the prevention of onset of labour or abortion was desired.

The opposite effect of inducing abortion by large doses of oestradiol has been attempted, and is generally considered ineffective, at least in the early stages of pregnancy. Large doses of oestradiol (Jeffcoate, I937), or of stilboestrol (Peel, I939) have been used with more success for inducing abortion after the intrauterine death of the foetus, or in the treatment of uterine inertia. In the latter condition, Jeffcoate used in one case 20,000 units of oestradiol spread over twelve hours, in another case $1,300,000$ units, spread over five days, in a third, 800,000 units spread over twelve hours, and in a fourth 700,000 units, spread over twelve hours. Reynolds (I939) reviews work published on the use of oestradiol in pregnancy. He concludes that it has not been proved of value in attempting to induce abortion with a live foetus or inducing premature labour. It does, however, appear to be of value in uterine inertia, and he quotes with approval the work of Jeffcoate and colleagues; and its most promising use appears to be in cases of intrauterine death, including missed abortion, as in such cases the dead foetus may be retained in the uterus for a considerable time if oestradiol is not employed. This is also important in so far as "the usual oxytocics are without effect in missed abortion, and if effective are not free from danger." 


\section{Pregnancy Toxaemia and Eclampsia}

An excellent review of the essential literature is to be found in the British Medical Journal (I942). Hofbauer (I9I8) first postulated an endocrine basis for eclampsia, and Kustner (I928) demonstrated that the blood of an eclamptic woman contained a substance which would stimulate expansion of melanophores in the frog. In I932 Anselmino, Hoffman and Kennedy drew attention to the fact that chronic pituitrin poisoning in rabbits produced pathological changes in the kidneys resembling those seen in pregnancy toxaemia. They also claimed to have demonstrated that, during toxaemia, there occurs an increased production of posterior pituitary hormones, vasopressin and anti-diuretic; and that as the clinical severity of the toxaemia increases, so can greater concentration of these hormones be demonstrated in the blood. These findings have led to much controversy and some contradiction. Cushing (I933) approached the problem from a histological angle, and showed that in fatal cases of eclampsia there was a basophilic hyperplasia of the pars intermedia, and often an infiltration of the pars nervosa by basophil cells. The significance of these findings is also disputed (Lennox, I942). Mukherjee (I94I) returned to the biological approach, and showed that filtrates from the blood of toxaemic women contained a melanophore-expanding principle, an antidiuretic substance, and a vasopressor substance, the relative proportion of the latter two constituents corresponding to the clinical picture.

Hain (I940) found that in normal women in the last three weeks of gestation a marked increase in the excretion of oestrogens and pregnanediol took place, and in pre-eclampsia or eclampsia the values for both were higher than in normal women, and especially high were the pregnanediol values. In one patient a sudden marked rise in pregnanediol followed intrauterine death. The complexity of the subject, however, is shown by the observations of Smith and Smith (I94I), who found that the earliest and most constant sign in pre-eclampsia and eclampsia was the elevation of serum gonadotrophins (implying a placental abnormality), and that there is a "progressive deficiency of progestin, a concomitant decrease of oestrogen, and a consequent changed metabolism of oestrogen, involving greater and more rapid destruction." Shute (I940) also found that the blood of eclamptic women revealed low oestrogen values, and records observation on the treatment of "ten true pre-eclamptics and six convulsive eclamptics to show the "beneficial effects of oestrogens in the treatment of these cases." $\mathrm{He}$ also warno against the giving of Vitamin $\mathrm{E}$ to eclamptic women, since this produces a still further lowering of the blood oestrogen values, and records that "one of the writer's patients developed a fulminating toxaemia ending in convulsions when large doses of wheat germ oil had been administered without the precaution of a preliminary test of blood oestrogen level"-and that "a colleague had two similar experiences." He adds that "there is a large gap in the chain of evidence." As to treatment, he gave 50,000 international units of oestradiol subcutaneously, and repeated this within twenty-four hours if no benefit. In some cases spontaneous labour supervened. Cohen, Marrian and Watson (I935), point out that normally "during the greater part of pregnancy over 99 per cent of the total oestrogenic material excreted in the urine is in the 'combined' ether-soluble form, which possesses only a low physiological potency. It is therefore no longer difficult to reconcile the production of large amounts of oestrogenic substances in the body during the pregnancy with the fact that the injection of oestrin into pregnant animals may interfere with the normal course of gestation."

It seems to the writer that a great deal of the abnormal hormone findings in eclampsia may be due to a diseased placenta, as this hormone is the source of oestrin, progestin, and gonadotropin; but that excess of vasopressin from a hypertrophied pituitary may produce vaso-spastic changes and resulting infarction in the placenta. On the whole one is tempted to agree with the cynical view that "eclampsia is a disease of theories."

\section{Pernicious Vomiting of Pregnancy}

Anker and Laland (I938) found high serum values and low urinary values of "anterior pituitary-like principles" in patients with pregnancy vomiting, as compared with uncomplicated pregnancy. Freeman and Melick (I935) found that extract of the suprarenal cortex was of value in pernicious vomiting of pregnancy, but put forward no theoretical consideration. Many remedies, however, have been advocated, for vomiting of pregnancy. In the early stages of pregnancy the commonest cause is psychological, but in the later stages of pregnancy pernicious

vomiting is probably a manifestation of "toxaemia of pregnancy." Anselimno (I936) states 
that with pernicious vomiting of pregnancy there is a disturbance of carbohydrate metabolism, an inability to store glycogen, and an increase in ketone bodies due to an increase in fat metabolism. He also observes that there is an absence of hypertrophy of the adrenal cortex in hyperemesis gravidum as compared with that which occurs in normal pregnancy. If this be so, it would constitute a rationale for therapy with adrenal cortical extracts. Synthetic desoxycorticosterone, however, should not be used, as it tends to produce salt-and-water retention. Corticosterone, the carbohydrate regulating factor in cortical extracts, is not available commercially as a pure synthetic preparation.

\section{Lactation}

Oestrogens and progesterone both influence mammary development, the former stimulating duct development, and the latter alveolar growth. However, both fail to act in a. hypophysectomised animal, whereas pituitary extracts produce breast development, the essential constituent being named "Mammogen." It is therefore postulated by Selye and Collip (I936) that ovarian hormones act on the mammary gland indirectly by way of their pituitary-stimulating effect. Mammogen is concerned with breast development, but another pituitary hormone "prolactin" is responsible for the initiation of lactation. Its secretion during pregnancy is prevented by the concentration of oestrogens in the blood, but when these fall at parturition prolactin is secreted and lactation begins. Should the pituitary mechanism not respond, lactation will not commence or will be inadequate. In such cases (but not in all cases of poor lactation) prolactin (e.g. Physolactin) may be injected intramuscularly, 5 c.c., 5 c.c., 2 c.c., 2 c.c. and I.c.c. on successive days, and lactation may thereby be induced or improved. Its maintenance depends upon the suckling reflex. When a child is stilll-born, it is necessary to suppress lactation, and this can be done by oestrogens or androgens, both inhibiting pituitary secretion. 50,000 units of oestradiol, or $5 \mathrm{mg}$. of stilboestrol or hexoestrol, may be injected I2-hourly, or 24-hourly, the dose being progressively reduced by each injection over a period of a few days; or $50 \mathrm{mg}$. androgens, repeated daily for a few days, is equally effective.

\section{Diabetes Mellitus}

This disorder should not be diagnosed unless. the reducing substance in the urine is known to be glucose (not lactose), and the tolerance curve is a characteristic diabetic one. Renal glycosuria, or diabetes innocens, associated with normal blood sugar values, and a low renal threshold for glucose, may occur during pregnancy, and is of no significance. Diabetes mellitus may start during pregnancy, and if slight may disappear after pregnancy, to recur at a successive pregnancy, or to become permanent later on. It seems probable that diabetes mellitus arising in pregnancy may be of pituitary origin, and due to the pituitary hyperplasia that occurs in pregnancy, and vitimately may become a pancreatic diabetes as in the experimental pituitary diabetes of dogs (Young, I939, Best and others, I939). It is therefore essential to treat even mild diabetes in pregnancy with care and thoroughness, because in this way a permanent diabetes may be prevented, just as diet or insulin may prevent experimental pituitary extract diabetes becoming permanent pancreatic diabetes.

Pregnancy does occur in diabetics, especially if well controlled by diet and insulin, although the fertility rate is lower than normal. When pregnancy does occur, the danger is greater to the foetus than to the mother. The causes of the high foetal mortality rate (some 40 per cent) are due mainly to the large size of the foetus, and to an increased incidence of toxaemias among diabetics (Smith, Smith, Joslin and White, I937), while other causes are post-natal hypoglycaemia, and perhaps a slightly increased incidence of congenital defects. Maternal mortality has fallen from 45 to 2 per cent since the introduction of insulin. The insulin requirement is normally raised during the second half of pregnancy, and falls again rapidly after delivery, when great care must be taken to avoid insulin hypoglycaemia. The ultimate requirements of insulin are not necessarily greater than before pregnancy. In diabetic women, as with normal women, the renal threshold is frequently lowered in pregnancy, so that blood sugar estimations are necessary as well as urine testing. The renal threshold is not a static level, and tends to its lowest level about the fifth month of pregnancy.

As regards foetal size, in 45 instances Lawrence and Oakley (I942) found that 27 babies weighed above 9 lbs., and 13 of these weighed between Io and 12 lbs. Caesarean section was performed in 27 cases, between the 36 th and 38 th week, and 23 of the babies survived; I9 births 
were spontaneous, and II babies survived; 3 were induced, and 2 survived. The total foetal mortality was 33 per cent. They conclude that pregnancy should always be terminated at the 36 th to the 38 th week, and favour Caesarean section in primipara and induction in multipara. This is in keeping with Joslin's experience. To avoid the possibility of insulin hypoglycaemia in the newborn baby (owing possibly to compensatory islet cell hypertrophy), glucose should be given two-hourly during the first forty-eight hours, although Joslin records that in the last $\stackrel{\rho}{9}$ twenty-five babies in his Clinic this has not been necessary. Lactation is often deficient in a diabetic, but breast feeding should be attempted whenever possible.

Priscilla White and Hazel Hunt, working at Joslin's Clinic, have recently made an interesting contribution to the study of foetal death rate in diabetes mellitus. They carried out assays of chorionic gonadotropin and pregnanediol in the urine of 125 diabetic women after the $24^{\text {th }}$ week of pregnancy. In 4I women these hormones were present in normal concentration, and 95 per cent of foetuses survived. In the remaining 84 cases, there was an increased excretion of gonadotropin and decreased excretion of pregnanediol. 27 of these abnormal patients were untreated by hormones and 60 per cent of the foetuses survived. 50 patients with abnormal hormone excretion were treated by large doses of progesterone, and stilboestrol, in the latter half or third of pregnancy, and the foetal survival rate was as high as 92 per cent. The authors also record that toxaemia appeared to be modified by this hormone therapy. If this work is confirmed, it would appear to call for the routine assay of chorionic gonadotropin and pregnanediol in pregnant diabetic women, as the apparent effect of appropriate hormone therapy on foetal death rate is striking.

As to whether diabetics should have children from a eugenic point of view, the answer is $\mathscr{C}^{\circ}$ in the negative if there is a family history of diabetes in both parents, and an indecisive answer 0 if only on one side of the family. Otherwise the answer is Yes, if the mother desires children and is intelligent. The Mendelian recessive theory is not universally accepted.

\section{Diabetes Insipidus}

This is a rare complication of pregnancy, and when it appears in pregnancy often disappear after partutition. A pre-existing diabetes insipidus may be aggravated, ameliorated, or unino. fluenced by pregnancy (Blotner and Kunkel, I942). The condition is readily controlled b $\vec{\not}$ injections of pitressin tannate in oil.

\section{Thyrotoxicosis}

This condition may have its onset in pregnancy, probably due to a pituitary thyrotropic stimulus in a patient with a constitutional thyroid sensitivity. More commonly pregnancy may supervene in a thyrotoxic patient, although with severe degrees of thyrotoxicosis pregnancy is unlikely, and if it occurs may be followed by spontaneous abortion. The treatment of pregmancy in a thyrotoxic patient used to be abortion in the early months, and this is justifiable. If, however, a child is desired, an early thyroidectomy is indicated and is likely to result both in the cure of the thyrotoxicosis and a successful pregnancy. It may be that the recent discovery of the beneficial effects of thiourea and thiouracil in thyrotoxicosis will permit the continuation of pregnancy under medical supervision, or in combination with irradiation of the thyroid gland. The maximum and most prompt safety for mother and foetus, however, is offered by thyroidectomy.

\section{Addison's Disease}

This condition, due to deficiency of the suprarenal glands, may have its onset in pregnancy, or pregnancy may supervene in a typical case of pre-existing Addison's disease. In the latter case, the adrenal insufficiency will be aggravated, but adequate treatment with cortical extract and desoxycorticosterone may permit a pregnancy to be continued successfully to full term. The risk to the mother, however, is considerable, and unless there are very strong reasons for attempting a difficult task, abortion should be performed in the first three months. Some patients with Addison's disease have a history of minor manifestation of the disorder in a previous pregnancy, with disappearance of the symptoms after parturition and onset of the disease proper some years later. In others, the disease commenced in pregnancy and continued subsequently. In both cases the adrenal glands were probably affected in slight degree by tuber- 
culosis, or idiopathic atrophy, before pregnancy supervened, and the manifestation of the adrenal insufficiency was brought about because of the extra demands made upon the adrenal cortex during pregnancy. It is probable that the pigmentation seen in some women during pregnancy is a manifestation of temporary adrenal insufficiency, as may be other features, e.g. gastro-intestinal disorders, asthenia and low blood pressure, and treatment with adrenal, cortical extract has appeared to be of benefit in such circumstances. If a therapeutic test is considered inadvisable, a Kepler diagnostic test for adrenal insufficiency may first be applied (Simpson, I94I).

\section{Acromegaly}

Mild acromegaly may occur in pregnancy, and may tend to persist. This is due to the physiological hypertrophy of the anterior pituitary gland overstepping normality in an individual with constitutional acromegaloid tendency. If pregnancy supervenes in an acromegalic woman, it should be allowed to run its normal course unless there is clinical evidence of severe aggravation of the acromegaly. Lactation may be excessive and prolonged in acromegaly, after weaning of the baby, but can be checked by oestradiol or testosterone. (See lactation.)

\section{Cushing's Syndrome}

This is a condition described by Cushing in I932, and characterised by hirsutism, amenorrhoea, adiposity, plethora, hypertension, glycosuria, and rarefaction of bones, etc.; and is associated with a basophil adenoma of the anterior pituitary gland, with hyalinisation of the remaining basophil cells, and/or neoplasm or hyperplasia of the adrenal cortex. It usually has nothing to do with pregnancy as the patients are sterile, but Cohen (I937) has described a case in which the syndrome appeared during pernicious vomiting of pregnancy and progressed to extreme degree. Spontaneous abortion was followed by a complete remission, and the whole process was repeated in the next pregnancy, when a further abortion led to another remission. This striking case lends support to the view that adiposity and mild hirsutism that not infrequently occur in pregnancy, with partial or complete remission after parturition, are manifestations of pituitary and adrenal gland changes beyond the physiological concomitants of pregnancy. The so-called "pregnancy cells" of the anterior pituitary gland are believed by Rasmussen to be eosinophil in origin, but it seems probable from the above considerations that the basophil cells are also increased, and all cellular elements of the anterior pituitary may be involved in pregnancy hyperplasia, their proportion or extent varying with the underlying endocrine constitution of the patient.

\section{Simmonds's Cachexia}

This disorder is not really a complication of pregnancy, except in so far as its cause may be due to something that happens at parturition. The condition was originally described by a Hamburg pathologist in a fatal case of puerperal fever, at the autopsy of which atrophy of the anterior lobe of the pituitary was found secondary to an arterial embolus. Sheehan and Murdoch (I938) point out that necrosis of a portion of the anterior lobe occurred more frequently than was realised as a result of thrombosis or embolus when parturition is associated with severe haemorrhage or infection, and that the syndrome may be incomplete and chronic. The classical features of Simmonds's cachexia are amenorrhoea, loss of secondary sexual characteristics, subnormal temperature and metabolism, loss of weight and bradycardia. I have recently seen a patient who developed the incomplete syndrome following a pregnancy at the age of thirty-two in the year I9I8. She had several attacks of severe and prolonged spontaneous hypoglycaemic coma, which responded to intravenous glucose, but the last attack (I943) was fatal, and at autopsy there was atrophy of the anterior pituitary and of the adrenal cortex (secondary to the pituitary). Thus the condition may be chronic over a period of twenty-five years. I have seen another case following parturition, which had been treated as myxoedema for many years, with a very incomplete response to thyroid gland, as might be expected (Simpson, I943). Thus care and skill at parturition and in the pre-natal supervision may prevent the onset of a very severe disorder which may prove fatal at once, or within a few months, or many years later, or that may lead to chronic invalidism. Whether adequate and immediate blood transfusions would prevent thrombosis of the pituitary vessels following a severe parturition haemorrhage 
it is difficult to know, but it is a problem that calls for consideration, and this might be preferable to awaiting a slower recovery from severe bleeding. There is much to be said in favour of immediate blood transfusion.

\title{
REFERENCES
}

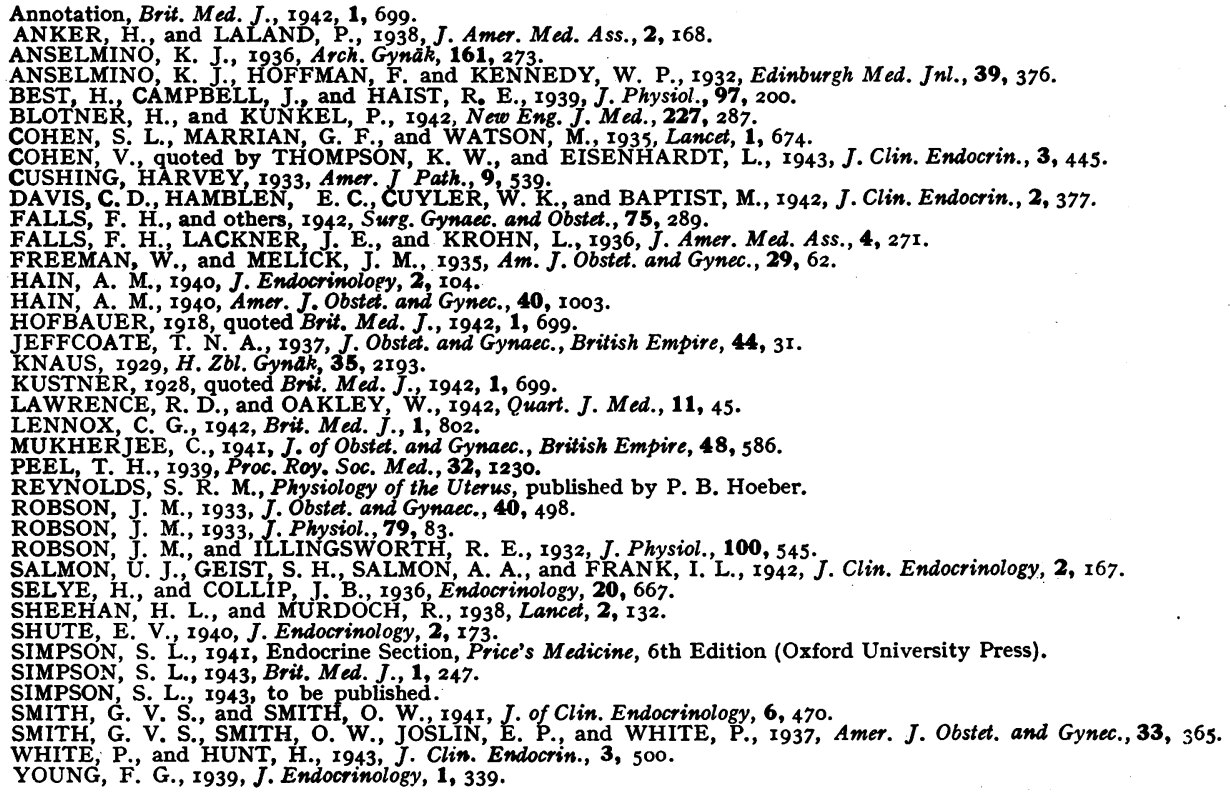

\section{BLOOD DISORDERS IN RELATION TO PREGNANCY}

\author{
By A. PINEY, M.D., M.R.C.P. \\ (Physician, St. Mary's Hospital, E.r3)
}

It is, of course, impossible, in the limits of a single article, to discuss all that is known of blood changes during pregnancy; and only those alterations that are recognised to be of practical significance will be considered. It is, however, timely to emphasise that our knowledge of the blood in normal and abnormal pregnancy is woefully deficient; and a great deal more observation is needed to fill the large gaps in our knowledge.

\section{Physiological changes.}

The development of the foetus, the increase in bulk of the uterus, the placental circulation, and the blood lost during parturition make considerable demands on the maternal stores of iron. Indeed, it has been estimated (Davidson and Fullerton), that these functions, together with the requirements of lactation, demand 900 milligrammes of iron.

This amounts to a need for a daily positive balance of 2 milligrammes of iron, which is not a great amount; and it is probable that hypochromic anaemia does not develop in pregnant women as a result of simple iron-deficiency directly due to uncomplicated pregnancy, if the state of health was previously good (see below). 